\title{
UNIQUE FACTORIZATION IN MODULES AND SYMMETRIC ALGEBRAS
}

\author{
BY
}

DOUGLAS L. COSTA

\begin{abstract}
Necessary and sufficient conditions are given for a torsionfree module $M$ over a UFD $D$ to admit a smallest factorial module containing it. This factorial hull is $\cap M_{P}$, the intersection taken over all height one primes of $D$. In case $M$ is finitely generated, the hull is $M^{* *}$, the bidual of $M$.

It is shown that if the symmetric algebra $S_{D}(M)$ admits a hull, then the hull is the smallest graded UFD containing $S_{D}(M) . S_{D}(M)$ is a UFD if and only if it is a factorial $D$-module. If $M$ is finitely generated over $D$, but not necessarily torsion-free, then $\bigoplus_{i \geqslant 0}\left(S^{i}(M)\right)^{* *}$ is a graded UFD.

Examples are given to show that any finite number of symmetric powers of $M$ may be factorial without $S_{D}(M)$ being factorial.
\end{abstract}

Introduction. Anne-Marie Nicolas defined and discussed factorial modules over unique factorization domains in [6], and in [7] extended this concept to that of factorable modules over arbitrary integral domains. A factorable module $M$ over an integral domain $D$ is a torsion-free module with the property that each nonzero element of $M$ may be written uniquely (up to units) as the product of an element of $D$ and an element of $M$ which is divisible only by units of $D$. A factorial module is a factorable module over a unique factorization domain. These have the property that elements of the module have unique factorizations.

Not every torsion-free module over a UFD may be embedded in a factorial module. We shall see, however, that if a torsion-free module $M$ is a submodule of a factorial module, then there is a unique smallest factorial module $\hat{M}$ containing $M$. In fact $\hat{M}=\bigcap_{P}$, where $P$ ranges over the principal prime ideals of $D$. We shall also relate the existence of $\hat{M}$ to some other conditions on $M$ and in the process arrive at an internal construction for $\hat{M}$.

Factorable modules were studied under the name "type 0 modules" in [2]. It was shown there that for $M$ torsion-free over a UFD $D$, the symmetric algebra $S_{D}(M)$ is a UFD if and only if it is a factorial $D$-module. Here we shall

Presented to the Society, November 7, 1975; received by the editors September $12,1975$. $15 \mathrm{A78}$.

AMS (MOS) subject classifications (1970). Primary 13C10, 13F15; Secondary

Key words and phrases. Factorial module, symmetric algebra. 
prove that if the module $\widehat{S_{D}(M)}$ exists, then it is a graded UFD, and in fact the smallest one containing $S_{D}(M)$ as a graded $D$-subalgebra.

Since the factoriality of $S_{D}(M)$ is equivalent to the factoriality of the symmetric powers $S_{D}^{i}(M)$ as $D$-modules, one might hope for some bound on the number of symmetric powers which must be checked. We shall conclude with a family of examples to dispel that hope.

Before proceeding we list here some other characterizations of factorial modules which we shall use freely in the sequel. For $D$ a UFD and $M$ a torsionfree $D$-module the following are equivalent:

(1) $M$ is factorial.

(2) Each nonzero element $x \in M$ has a greatest divisor $d \in D$, in the sense that any other divisor of $x$ must be a factor of $d$. (We shall use g.d.(x) to denote the greatest divisor of $x$.)

(3) Every maximal rank one submodule of $M$ is cyclic [6, Theorem 8.1], [7, Theorem 3.7].

(4) $M$ satisfies the ascending chain condition on cyclic submodules (a.c.c.c.), and every $D$-sequence of two elements is an $M$-sequence [6, Theorem 1.7, Proposition 4.1, Proposition 4.5].

If $M$ is factorial, elements of $M$ which are divisible only by units of $D$ are called primitive. Each nonzero element $x \in M$ has the unique representation $x=\operatorname{g.d} .(x) x^{*}$, where $x^{*}$ is primitive.

1. Let $D$ be a UFD with quotient field $K$. Let $M$ be a torsion-free $D$. module and set $K M=K \otimes_{D} M$.

1.1. Definition. A $D$-module $\hat{M}$ is a factorial hull of $M$ if $M \subseteq \hat{M}, \hat{M}$ is factorial, and $M \subseteq \hat{M} \subseteq N$ whenever $M \subseteq N$ with $N$ factorial.

If a factorial hull exists, it is unique and so may be referred to as the factorial hull. Not every torsion-free module has a factorial hull. The quotient field $K$, for example, is a maximal rank one submodule of any module in which it sits, but is cyclic only if $D=K$.

1.2. Proposition. If $\left\{M_{i} \mid i \in I\right\}$ is a family of factorial submodules of a torsion-free $D$-module $M$, then $\bigcap_{M_{i}}$ is factorial.

Proof. $\cap M_{i}$ is torsion-free. Let $x \in \bigcap M_{i}$. For each $i \in I$ write $x=$ $d_{i} x_{i}^{*}$, with $d_{i} \in D$ and $x_{i}^{*}$ primitive in $M_{i}$. Let $d=$ g.c.d. $\left\{d_{i} \mid i \in I\right\}$. Then $x=d c_{i} x_{i}^{*}$ for each $i \in I$, and since $M$ is torsion-free, $c_{i} x_{i}^{*}=c_{j} x_{j}^{*}$ for $i, j \in I$. Thus $x / d \in \bigcap M_{i}$. If $x / c \in \bigcap M_{i}, c \in D$, then $c \mid d_{i}$ for every $i \in I$ and hence $c \mid d$. This shows that $d$ is a greatest divisor for $x$.

1.3. THEOREM. If $M$ is a submodule of a factorial module $N$, then there is a smallest factorial submodule $\hat{M}$ of $N$ containing $M . \hat{M}$ is a factorial hull of M. Furthermore, $\hat{M} \subseteq K M$. 
PROOF. The proposition shows that the intersection of the family of factorial submodules of $N$ containing $M$ is factorial, proving the first assertion. Now suppose $M \subseteq L$ with $L$ a factorial $D$-module. (Note that we may take $L$ $=N$.) Construct the push-out

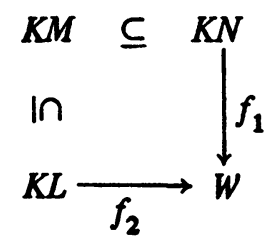

where $W$ is the vector space $K L \oplus K N /\{(x,-x) \mid x \in K M\}, f_{1}(y)=\overline{(0, y)}$, and $f_{2}(z)=\overline{(z, 0)}$. Then $f_{1}, f_{2}$ are injective and the diagram commutes. So we may consider $M, L, N, K M, K L, K N$ to be submodules of $W$, with all containment relations holding there. Since $W$ is torsion-free, $L \cap N$ is a factorial submodule of $N$, whence $\hat{M} \subseteq L \cap N \subseteq L$. Now also $\hat{M} \subseteq L \cap N \subseteq K L \cap K N=K M$ by construction.

1.4. COROLlARY. Every finitely generated torsion-free module over a UFD has a factorial hull.

Proof. Every such module is contained in a free module, and these are all factorial [6, Theorem 3.2].

From now on $\hat{M}$ will denote the factorial hull of $M$.

1.5. Proposition. If $\hat{M}$ exists, then $\hat{M}=\bigcap\left\{M_{P} \mid P\right.$ is a height one prime of $D\}$.

PROOF. First note that for any torsion-free module $N$ over the UFD $D$, $N=\bigcap\left\{N_{P} \mid \mathrm{ht}(P)=1\right\}$ if and only if every $D$-sequence of length 2 is an $N$. sequence. $\cap M_{P}$ clearly has this property and, since it is factorial, $\hat{M}$ does too. Now $M_{P} \subseteq(\hat{M})_{P}$ for every height one prime $P$, so $\cap M_{P} \subseteq \hat{M}$. Since $\hat{M}$ is factorial, $\cap M_{P}$ inherits from it the a.c.c.c. It follows that $\cap M_{P}$ is factorial and therefore that $\hat{M}=\bigcap M_{P}$.

1.6. COROLlARY. If $M$ is finitely generated, then $\hat{M}=M^{* *}$, the bidual cf $M$.

Proof. This follows from $\bigcap M_{P}=M^{* *}[1]$.

It is by now clear that $M$ has a factorial hull if and only if $M$ is a submodule of a factorial module if and only if $\bigcap M_{P}$ is factorial. We wish to characterize those torsion-free modules which admit a factorial hull, i.e., those for which $\bigcap M_{P}$ is factorial. In order to do so it is convenient to introduce the 
following notion. Suppose that $\left\{D_{i} \mid i \in I\right\}$ is a family of integral domains with the same quotient field $K$, and that, for each $i \in I, M_{i}$ is a factorable $D_{i}$-submodule of some vector space $V$ over $K$. We shall say that the intersection $\cap M_{i}$ is locally finite if each nonzero $x \in \bigcap M_{i}$ is primitive in all but a finite number of the $M_{i}$ 's.

1.7. Proposition. Let $M$ be a torsion-free module over a UFD $D$. $\bigcap\left\{M_{P} \mid\right.$ ht $\left.(P)=1\right\}$ is factorial if and only if each $M_{P}$ is a factorial $D_{P}$-module and the intersection is locally finite.

Proof. We may assume that $M=\bigcap M_{P}$. If $M$ is factorial, then, for each prime ideal $P, M_{P}$ is factorial as a $D_{P}$-module and for each nonzero element $x \in M$, g.d. ${ }_{D_{P}}(x)=$ g.d.(x) [2, Proposition 2.20]. (g.d. ${ }_{D_{P}}(x)$ is the greatest divisor in $D_{P}$ of the element $x \in M_{P}$.) Thus g.d. ${ }_{D_{P}}(x)$ is a unit in $D_{P}$ for all but a finite number of height one primes $P$. It follows that $\bigcap M_{P}$ is locally finite.

Conversely, if $M=\bigcap M_{P}$ is a locally finite intersection of factorial modules, for any nonzero $x \in M$ and any prime $p \in D$ we may write g.d. ${ }_{(p)}(x)=p^{n_{p}}$ with $n_{p} \geqslant 0$. Then $n_{p}=0$ for all but a finite number of nonassociate primes $p$. Let $d=\Pi p^{n} p$. Since $x / d \in M_{P}$ for each height one prime $P, x / d \in M$. If also $x / c \in M$, then $x / c \in M_{(p)}$ for each prime $p \in D$ and so $c \mid p^{n^{p}}$ in $D_{(p)}$. Therefore $c \mid d$, so that $d$ is a greatest divisor for $x$. This shows that $M$ is factorial.

We may extract from the preceding proposition an internal characterization of those modules which have factorial hulls.

1.8. LEMMA. Let $M$ be a torsion-free module over a UFD D. M has a factorial hull if and only if for each nonzero $x \in M$ there is a nonzero element $d \in D$ such that for any $a \in D$ the divisors of ax must divide ad.

Proof. Suppose that $\hat{M}$ exists and let $x \in M$ be nonzero. Then $x$ has a greatest divisor $d \in D$, as an element of $\hat{M}$. If $c$ is a divisor of $a x$ in $M$, then it is a divisor of $a x$ in $\hat{M}$ and so $c \mid$ g.d. $(a x)=a d$.

Conversely, let $x \in M$ be nonzero and let $d \in D$ be the element guaranteed by the hypothesis. Let $p \in D$ be a prime. If for some positive integer $n, p^{n}$ divides $x$ in $M_{(p)}$, then $p^{n}$ divides $t x$ in $M$ for some $t \in D \backslash(p)$. This forces $p^{n} \mid t d$ and therefore $p^{n} \mid d$. It follows that $n$ is bounded and that $M_{(p)}$ is a factorial $D_{(p)}$-module. It also follows that $x$ is primitive in $M_{(p)}$ for all but a finite number of $(p)$. By the proposition, $\hat{M}$ exists.

We conclude this section with two simple examples.

(1) Let $D$ be a UFD which is not a PID, and let $M$ be any ideal of $D$ of height at least two. Then $M$ is not a principal ideal and is therefore not a 
factorial module. $\cap M_{P}=D$ is factorial, however, so that $\hat{M}=D$.

(2) Let $D$ be any UFD and $M$ the submodule of its quotient field consisting of all fractions with square-free denominators. For each prime $p \in D$, $M_{(p)}=(1 / p) D_{(p)}$ is factorial, and $\cap M_{P}=M$. The intersection is not locally finite, however, since $1 \in M$ is divisible by every prime.

2. The finite divisor property. We shall say that a torsion-free module over a UFD has the finite divisor property (f.d.p.) if each nonzero element of the module has, up to units, only a finite number of proper divisors. Equivalently, a module has the f.d.p. if and only if each nonzero element is divisible by only a finite number of primes and only by a finite power of each. (One should perhaps define a notion of type as in $[4, \S 85]$, and call modules with the f.d.p. "homogeneous of type $(\ldots, 0,0, \ldots)$ ".)

Consider the following conditions on a torsion-free module $M$ over a UFD D:

(a) $M$ is factorial.

(b) $M$ has a factorial hull.

(c) $M$ has the f.d.p.

(d) $M$ satisfies the a.c.c.c.

In this section we hope to shed some light on the relations between these conditions. Some conclusions are immediate. First, it is clear that (a) implies (b) implies (c) implies (d). Second, if $M$ is flat, every $D$-sequence of length two is an $M$-sequence; hence (a), (b), (c) and (d) are equivalent for flat modules. In particular, all are equivalent over a PID. Third, we have already seen an example in $\$ 1$ to show that (a) and (b) are not equivalent in general.

For any torsion-free module $M$ over a UFD $D$, we may construct an enlargement of $M$ in the following manner. For every prime $p \in D$ and every $x \in$ $M$ let $o_{p}(x)=\sup \left\{n \mid x \in p^{n} M\right\}$. We allow $o_{p}(x)=\infty$. Note that $o_{p}(x+y) \geqslant$ $\inf \left\{o_{p}(x), o_{p}(y)\right\}$; and for $d \in D, o_{p}(d x) \geqslant v_{p}(d)+o_{p}(x)\left(v_{p}\right.$ is the $p$-adic valuation on $D)$. Set $\widetilde{M}=\left\{x / d \in K M \mid x \in M, d \in D, d \neq 0\right.$ and $v_{p}(d) \leqslant o_{p}(x)$ for every prime $p \in D\}$. Then $M \subseteq \widetilde{M} \subseteq K M$ and $\widetilde{M}$ is a $D$-module. If $M$ has the finite divisor property, then $\widetilde{M}$ consists of all fractions $x / d$, where $d$ is a divisor of the least common multiple of the (finite) set of divisors of $x$.

2.1. LEMMA. Let $M$ be torsion-free over the UFD D. Then $\tilde{M}=$ $\bigcap\left\{M_{P} \mid\right.$ ht $\left.P=1\right\}$.

Proof. Let $x \in M$ and $d \in D$ be such that $v_{p}(d) \leqslant o_{p}(x)$ for every prime $p \in D$. Then for any prime $p, p^{v_{p}(d)}$ is a divisor of $x$. It follows that $x / d \in$ $M_{(p)}$. This shows that $\tilde{M} \subseteq \bigcap M_{P}$.

Next let $z \in \bigcap M_{P}$ and write $z=x / d$ with $x \in M$ and $d \in D$. Let 
$p_{1}, \ldots, p_{n}$ be those primes which divide $d$. For $1 \leqslant i \leqslant n$ we may write $x / d$ $=x_{i} / d_{i}$, where $p_{i} \nmid d_{i}$. Let $c=d_{1} \cdots d_{n}$ and $c_{i}=d_{1} \cdots \hat{d}_{i} \cdots d_{n}$. Then $c x=d c_{i} x_{i}$, for each $i$, and so $o_{p_{i}}(c x)=o_{p_{i}}\left(d c_{i} x_{i}\right) \geqslant v_{p_{i}}\left(d c_{i}\right)+o_{p_{i}}(x) \geqslant$ $v_{p_{i}}\left(d c_{i}\right)=v_{p_{i}}(c d)$. If $p$ is a prime which does not divide $d$, then $o_{p}(c x) \geqslant v_{p}(c)$ $=v_{p}(c d)$. Hence $x / d=c x / c d \in \tilde{M}$.

From the lemma it is clear that $\widetilde{M}=\widetilde{M}$. The lemma, together with the results of $\S 1$, also yields the following corollary.

2.2. Corollary. Let $M$ be a torsion-free module over a UFD. The following are equivalent.

(1) $M$ has a factorial hull.

(2) $\widetilde{M}$ is factorial.

(3) $\widetilde{M}$ has the f.d.p.

(4) $\widetilde{M}$ satisfies the a.c.c.c.

Moreover, if any of these conditions hold, then $\tilde{M}=\hat{M}$.

We can now give an example to show that (c) does not imply (b).

2.3. EXAMPLE. A module with the f.d.p. which has no factorial hull. Let $D$ be a noetherian UFD which is not a PID. Let $p, q$ be primes of $D$ such that $D p+D q \neq D$. Then $D[q / p]$ consists of the elements in the quotient field of $D$ of the form $\sum_{i=0}^{n} a_{i} p^{n-i} q^{i} / p^{n}$. Note that each such element is either in $D$ or may be written in the above form with $n>0$ and $p \nmid a_{n}$.

Consider the $D$-module $D[q / p]$. For $m \geqslant 0, q^{m}$ is divisible by both $q^{m}$ and $p^{m}$. Hence $1 / p^{m}=q^{m} / p^{m} q^{m} \in \widehat{D[q / p]}$, for $m>0$. This shows that $D[q / p]$ has no factorial hull.

Next we want to see that $D[q / p]$ has the f.d.p. To check this it is sufficient to check that each nonzero element in $D$ (considered as an element of the module $D[q / p])$ has a finite number of divisors, since for $x \in D[q / p]$ and a suitably chosen $N, p^{N} x \in D$. Therefore, let $d \in D, d \neq 0$, and let $r$ be a prime of $D$ distinct from $p$. If $d / r^{m} \in D[q / p]$, then either $d / r^{m} \in D$ or $d / r^{m}=$ $a / p^{n}$ with $n>0$ and $p \nmid a$. In the latter case $r^{m} a=p^{n} d$ implies $r^{m} \mid d$. Hence the only prime power divisors of the module element $d$ are the prime powers occurring in the factorization of $d$ and, possibly, powers of $p$. We will be done as soon as we see that $d$ is not divisible by arbitrarily large powers of $p$. This follows from the ensuing lemma.

Lemma. Let $D$ be a UFD and $p, q$ primes of $D$. A nonzero element $d \in D$ is divisible by arbitrarily large powers of $p$ in $D[q / p]$ if and only if $d \in$ $\bigcap_{n>0}(p, q)^{n} D$.

PRoof. Let $d \in D$ and suppose $p \nmid d$. If $d / p^{N} \in D[q / p]$, then we may 
write $d / p^{N}=\sum_{i=0}^{n} a_{i} p^{n-i} q^{i} / p^{n}$ with $n>0$ and $p \nmid a_{n}$. Then from $p^{n} d=$ $p^{N} \sum_{i=0}^{n} a_{i} p^{n-i} q^{i}$ we deduce that $n=N$ and $d \in(p, q)^{N} D$. This is enough to prove the necessity.

Conversely, if $d \in(p, q)^{N} D$, then $d=\Sigma_{i=0}^{N} a_{i} p^{n-i} q^{i}$ and hence $d / p^{N} \in$ $D[q / p]$.

We conclude this section with an example to show that (d) does not imply (c).

2.4. EXAMPLE. A module satisfying the a.c.c.c. which does not have the f.d.p. Let $D$ be a two-dimensional local UFD and $\underline{m}$ its maximal ideal. Then $\bigcap_{k>0} \underline{m}^{k}=0$ and $\underline{m}$ contains an infinite number of distinct primes of $D$. Furthermore, we may choose $D, \underline{m}$ so that $\underline{m}$ contains a sequence $\left\{p_{i}\right\}_{i=1}^{\infty}$ of distinct primes, each having the property that if $x \in \underline{m}^{k} \backslash \underline{m}^{k+1}$, then $p_{i} x \in \underline{m}^{k+1} \backslash \underline{m}^{k+2}$. (A polynomial ring in two variables over an infinite field localized at the origin will suffice.)

Let $M$ be the $D$-module generated by the fractions $1 / p_{i}, i \geqslant 1$. Since $1 \in M$ is divisible by every $p_{i}, M$ does not have the f.d.p. If $D x_{1} \subseteq D x_{2} \subseteq \cdots$ is an ascending chain of cyclic submodules of $M$, then $x_{1}=c_{1} x_{2}=c_{1} c_{2} x_{3}=\cdots$, where each $c_{i}$ is a nonunit of $D$. Let $x_{1}=\sum_{i=1}^{n} d_{i} / p_{i}=d / p_{1} \cdots p_{n}$. Since we may assume that $x_{1} \neq 0$, there is a positive integer $k$ such that $d \in$ $\underline{m}^{k} \backslash \underline{m}^{k+1}$. Now $x_{1} / c_{1} \cdots c_{k+1}=x_{k+2} \in M$ and we may write $x_{k+2}=$ $\Sigma_{i=1}^{N} a_{i} / p_{i}=a / p_{1} \cdots p_{N}$, where $a \in \underline{m}^{N-1}$. Then $d p_{1} \cdots p_{N}=a c_{1} \cdots$ $c_{k+1} p_{1} \cdots p_{n}$ yields the contradiction that the left-hand side is in $\underline{m}^{k+N} \backslash \underline{m}^{k+N+1}$, while the right-hand side is in $\underline{m}^{k+N+n} \subseteq \underline{m}^{k+N+1}$. This shows that $M$ satisfies the a.c.c.c.

3. Applications to symmetric algebras. We first need some lemmas.

3.1. Lemma. Let $D$ be $a$ UFD and $M=\bigoplus_{i \in I} M_{i}$ a torsion-free $D$ module. $\hat{M}$ exists if and only if $\hat{M}_{i}$ exists for each $i \in I$, in which case $\hat{M}=$ $\bigoplus_{i \in I} \hat{M}_{i}$.

Proof. This follows easily from the fact that a direct sum is factorial if and only if each summand is factorial [6, Theorem 3.2].

3.2. Lemma. Let $D=\bigoplus_{n>0} D_{n}$ be a graded domain. Then $D$ satisfies the ascending chain condition on principal ideals (a.c.c.p.) if and only if $D$ satisfies the a.c.c.c. as a $D_{0}$-module.

Proof. Routine.

If $D_{0} \subseteq D$ are integral domains we say that $D_{0}$ is inert in $D$ if, for $a, b \in$ $D, a \neq 0 \neq b, a b \in D_{0}$ implies that $a, b \in D_{0}$. Observe that if $D$ is a graded 
domain with $D_{0}$ its elements of degree zero, then $D_{0}$ is inert in $D$.

3.3. Lemma. Let $D_{0} \subseteq D$ be integral domains with $D_{0}$ inert in $D$. If $D$ is a UFD, then $D_{0}$ is a UFD and $D$ is a factorial $D_{0}$-module.

Proof. It is well known that if $D$ is a UFD, then so is $D_{0}$ and every prime of $D_{0}$ is a prime of $D$. For $x \in D, x \neq 0$, write $x=p_{1} \cdots p_{s} \cdot q_{1}$ $\cdots q_{t}$, where the $p_{i}$ 's are primes of $D_{0}$ and the $q_{j}$ 's are primes of $D$ not in $D_{0}$. Then $p_{1} \cdots p_{s}$ is a greatest divisor for $x$.

We are now ready for the main result of this section.

3.4. Theorem. Let $D$ be a UFD and $M$ a D-module such that the symmetric algebra $S_{D}(M)$ is torsion-free over $D$. If $S_{D}(M)$ exists, then it is a graded UFD.

Proof. First observe as in [8] that $S_{D}(M)$ is a subring of the polynomial ring $S_{k}(K M)$, where $K$ is the quotient field of $D$, and hence that $S_{D}(M)$ is a domain. As $S_{D}(M)=\bigcap_{\text {ht } P=1}\left(S_{D}(M)\right)_{D \backslash P}=\bigcap_{\mathrm{ht} P=1} S_{D_{P}}\left(M_{P}\right)$, it is a graded domain. Furthermore $S_{D}(M)_{D \backslash 0}=S_{K}(K M)$ is a UFD. Since $S_{D}(M)$ is a factorial $D$-module, it satisties the a.c.c.p. by Lemma 3.2. Using Nagata's theorem, it now suffices to show that the multiplicative system $D \backslash 0$ is generated by primes i.e., that each prime of $D$ is prime in $\widehat{S_{D}(M)}=T$. Let $p \in D$ be prime and let $P=p D$. Let $x, y, z \in T$ and suppose that $x y=p z$. This relation remains true in $T_{P}=S_{D_{P}}\left(M_{P}\right)$. Note that if $N$ is a finitely generated $D_{P}$-submodule of $M_{P}$, it is free and hence, by [5, Corollary 3.12], $S_{D_{P}}(N) \subseteq T_{P}$. Choose such an $N$ so that $x, y, z \in S_{D_{P}}(N)$. Since $S_{D_{P}}(N)$ is a polynomial ring over $D_{P}$ and $p$ is a prime, $p \mid x$ or $p \mid y$ there and hence in $T_{p}$. Say $p \mid x$ in $T_{p}$. Then there is a $d \in D \backslash P$ and a $t \in T$ such that $d x=p t$. Since $T$ is a factorial $D$-module, we must have $p \mid x$ in $T$. Thus $p$ is prime in $T$.

Note that if $D^{\prime}$ is a graded UFD over $D$ which contains $S_{D}(M)$ as a graded $D$-subalgebra, then since $D^{\prime}$ is a factorial $D$-module (Lemma 3.3) we have $\widehat{S_{D}(M)} \subseteq D^{\prime}$. Thus $S_{D}(M)$ is the smallest graded UFD containing $S_{D}(M)$ as graded $D$-subalgebra. A UFD containing $D$ which is also a factorial $D$-module is called a factorial extension of $D$ in [7]. $S_{D}(M)$ is clearly the smallest factorial extension of $D$ containing $S_{D}(M)$.

Let us now derive some corollaries to the theorem.

3.5. Corollary [2, Theorem 2.16]. Let $R$ be a ring and $M$ an $R$-module. $S_{R}(M)$ is a UFD if and only if $R$ is a UFD and $S_{R}(M)$ is a factorial $R$-module.

Proof. Necessity follows from Lemma 3.3. Sufficiency follows from the theorem. 
3.6. Corollary. Let $D$ be a UFD and $M$ a flat $D$-module. Then $S_{D}(M)$ is a UFD if and only if $S_{D}(M)$ satisfies the a.c.c.p.

Proof. As $M$ is flat, so is $S_{D}(M)$ [5, Proposition 2.3]. It now follows from 3.5 and the discussion in $\$ 2$ that $S_{D}(M)$ is a UFD if and only if it satisfies the a.c.c.c. as a $D$-module. Applying Lemma 3.2 finishes the proof.

3.7. Corollary. Let $M$ be a finitely generated module over a UFD $D$. Then $\bigoplus_{i>0}\left(S_{D}^{i}(M)\right)^{* *}$ is a graded UFD.

Proof. For any $D$-module $N$ let $t(N)$ be its torsion submodule. It is easy to check that $\operatorname{Hom}(N, D) \cong \operatorname{Hom}(N / t(N), D)$ and hence that $N^{* *} \cong$ $(N / t(N))^{* *}$. Thus

$$
\bigoplus_{i>0}\left(S^{i}(M)\right)^{* *} \cong \bigoplus_{i \geqslant 0}\left(S^{i}(M) / t\left(S^{i}(M)\right)\right)^{* *}=\bigoplus_{i>0}\left(S^{i}(M) / t\left(S^{i}(M)\right)\right),
$$

using the fact that $M$ is finitely generated, Corollary 1.6, and Lemma 3.1. Let $T=\bigoplus_{i \geqslant 0}\left(S^{i}(M) / t\left(S^{i}(M)\right)\right)=S(M) / t(S(M))$. Since $T$ is the canonical image of $S(M)$ in $S(M) \otimes K=S_{K}(M \otimes K)$, it is a graded domain. ( $K=$ quotient field of $D$.) We wish to see that $\hat{T}$ is a graded UFD.

Toward this end, observe that there is a commutative diagram of graded D-algebras,

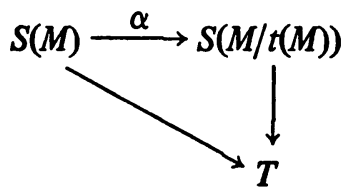

in which $\alpha$ is surjective. Applying the functor $F(\cdot)=(\cdot) / t(\cdot)$ to this diagram yields

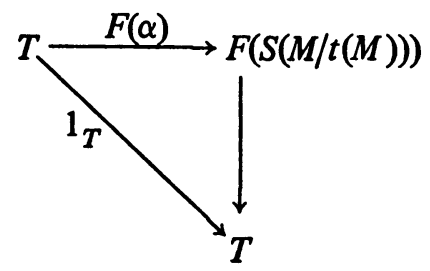

where $F(\alpha)$ is an isomorphism. It follows that the image of $S(M / t(M))$ in $S_{K}(M \otimes K)$ is $T$. Thus we may assume that $M$ is torsion-free.

Now for each height one prime $P$ of $D, M_{P}$ is free and hence $S(M)_{P} \subseteq$ $S_{K}(K M)$. Then $T \subseteq S(M)_{P}$ and since $T$ is the image of $S(M)$ in $S(M)_{P}$, we have $T_{P}=S(M)_{P}$. Thus $\hat{T}=\bigcap_{\mathrm{ht} P=1} S(M)_{P}$ is a graded domain over $D$. Now $\hat{T} \otimes K=S_{K}(K M), \hat{T}_{P}=S_{D_{P}}\left(M_{P}\right)$ for each height one prime of $D$, and $\hat{T}$ is a 
factorial $D$-module. Proceeding as in the proof of Theorem 3.4 proves that $\hat{T}$ is a UFD.

Note that if in Corollary 3.7 we had initially assumed $S_{D}(M)$ to be torsionfree, we would have had $\bigoplus\left(S_{D}^{i}(M)\right)^{* *}=\widehat{S_{D}(M)}$, and 3.7 would have been an immediate consequence of Theorem 3.4. Since in this setting $S_{D}(M)$ is an integral domain, one wonders whether $S_{D}(M)$ coincides with the integral closure of $S_{D}(M)$. That it does not can be seen as follows. Let $D_{0}$ be any UFD and let $X, Y, Z, U, V$ be indeterminates over $D_{0}$. Set $D=D_{0}[X, Y]$ and let $M$ be the ideal generated by $X$ and $Y$. Since $M \cong D \oplus D /\langle(Y,-X)\rangle, S_{D}(M) \cong$ $D[U, V] /(Y U-X V)$ which is a Krull domain by [3, Proposition 14.5]. Since $M$ is of rank one but not cyclic, it is not factorial, so neither is $S_{D}(M)$. In fact $S_{D}(M)=\bigcap_{\text {ht } P=1} S_{D_{P}}\left(M_{P}\right)=\bigcap_{\text {ht } P=1} S_{D_{P}}\left(D_{P}\right)=S_{D}(D) \cong D[Z]$. (Cf. the discussion at the end of [8].)

38. Proposition. Let $D$ be $a U F D$ and $M$ a finitely generated $D$-module. $S_{D}(M)$ is a UFD if and only if

(i) $S_{D}(M)$ is an integral domain, and

(ii) $S_{D / p D}(M / p M)$ is an integral domain for each prime $p$ of $D$.

Proof. If $S_{D}(M)$ is a UFD (i) clearly holds; and since $D$ is inert in $S_{D}(M)$, $S_{D / p D}(M / p M)=S_{D}(M) / p S_{D}(M)$ is a domain for each prime $p$ of $D$.

Conversely, if (i) and (ii) hold, then $S_{D}(M)$ is a domain and every prime of $D$ is prime in $S_{D}(M)$. Also, since $S_{D}(M)$ exists, $S_{D}(M)$ satisfies the a.c.c.c. as a $D$-module, and hence the a.c.c.p. as a ring. By Nagata's theorem $S_{D}(M)$ is a UFD.

Proposition 3.8 has the effect, for finitely generated modules, of reducing the problem of the factoriality of symmetric algebras to the problem of their integrity. The problem of integrity is a difficult one. Samuel [8] made the observation that $S_{D}(M)$ is a domain if and only if it is torsion-free, but there is no necessary and sufficient condition on $M$ for $S_{D}(M)$ to be a domain. Any such condition would have to logically fall strictly between the properties of flatness and torsion-freeness, as examples show.

Proposition 3.8 can be reformulated in the following way.

38'. Proposition. Let $D$ be a UFD; $X_{1}, \ldots, X_{n}$ indeterminates over $D$; and $I$ an ideal of $D\left[X_{1}, \ldots, X_{n}\right]$ generated by linear forms. Then $D\left[X_{1}, \ldots, X_{n}\right] / I$ is a UFD if and only if $I$ is prime and $(p, I)$ is prime for every prime $p \in D$.

4. A family of examples. In [7, Example 6.3] Nicolas constructs an example of a rank two torsion-free abelian group which is a factorial module over 
the integers, but which is not free or even a submodule of any direct product of copies of the integers. We are going to use the same construction with a few modifications which will enable us to draw conclusions about the symmetric algebra of the resulting module.

Let $D$ be an integral domain with quotient field $K$. Let $\left\{p_{i}\right\}_{i=1}^{\infty}$ be a sequence of nonzero elements of $D$. Inductively define a sequence of rank two free $D$-submodules of $K \oplus K$ as follows. Let $F_{1}=D \oplus D$, and once $F_{1}, \ldots, F_{n}$ have been chosen, choose $F_{n+1}$ so that the matrix of the inclusion map $F_{n} \subseteq$ $F_{n+1}$ is

$$
\left(\begin{array}{cc}
1 & 0 \\
-1 & p_{n}
\end{array}\right)
$$

(This is possible since the matrix has nonzero determinant.) Set $M=\bigcup_{i=1}^{\infty} F_{i}$. We shall refer to $M$ as the module determined by the sequence $\left\{p_{i}\right\}$. Note that $M$ is flat of rank two and is free if and only if it is finitely generated if and only if all but a finite number of the $p_{i}$ 's are units.

Since formation of symmetric algebras commutes with direct limits, $S(M)$ $=\lim S\left(F_{i}\right)$. But by [5, Corollary 3.12] we have $S\left(F_{1}\right) \subseteq S\left(F_{2}\right) \subseteq \cdots$ and hence $S(M)=\bigcup_{i=1}^{\infty} S\left(F_{i}\right)$. Now $S\left(F_{i}\right)=D\left[X_{i}, Y_{i}\right]$ for each $i$, where $X_{i}$ and $Y_{i}$ are indeterminates. Furthermore we know that $X_{1}=X_{i+1}$ and $Y_{i}=-X_{i+1}$ $+p_{i} Y_{i+1}$ for each $i \geqslant 1$. Since, for $m \geqslant 0$, the symmetric power $S^{m}\left(F_{i}\right)$ is the free $D$-module with basis $X_{i}^{m}, X_{i}^{m-1} Y_{i}, \ldots, Y_{i}^{m}$, the equations in the preceding sentence allow us to compute the matrix for the inclusion map $S^{m}\left(F_{i}\right) \subseteq$ $S^{m}\left(F_{i+1}\right)$. The matrix is the lower triangular $m+1$ by $m+1$ matrix

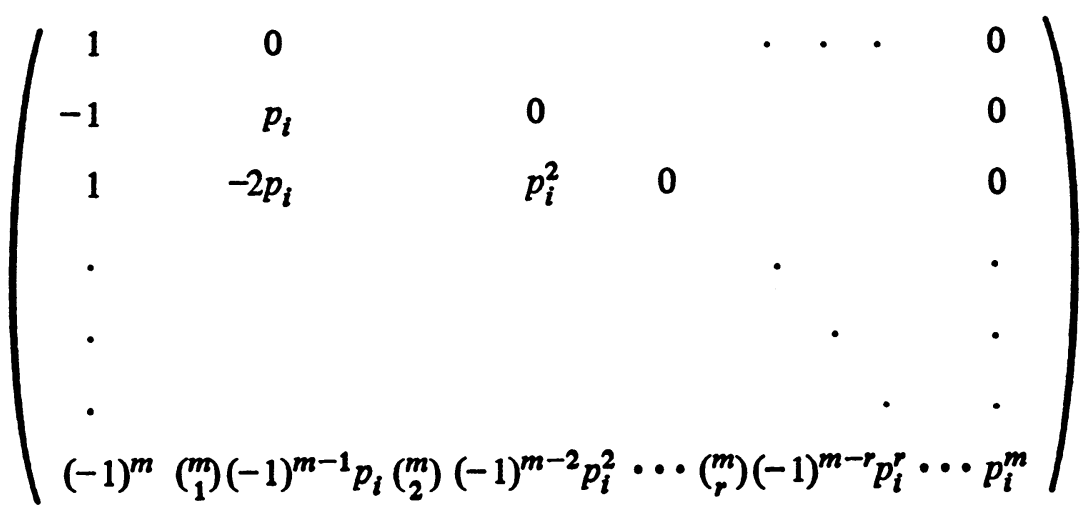

By induction one then shows that the matrix for the inclusion map $S^{m}\left(F_{1}\right)$ $\subseteq S^{m}\left(F_{n+1}\right)$ is the lower triangular $m+1$ by $m+1$ matrix 
D. L. $\cos T A$

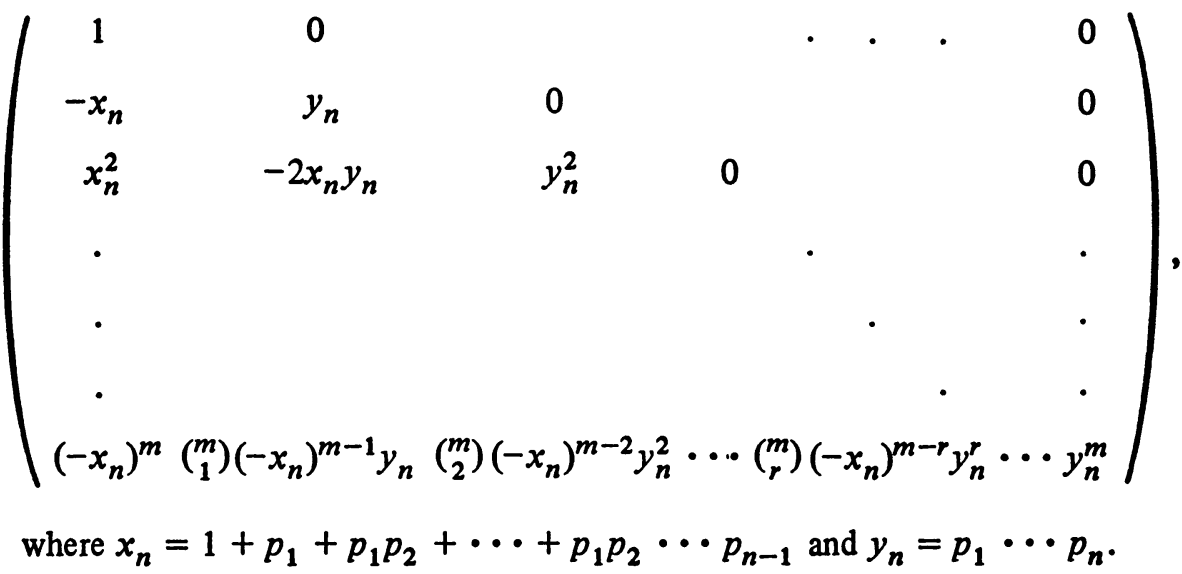

(Note that $x_{n+1}=x_{n}+y_{n}$ and $y_{n+1}=p_{n+1} y_{n}$.)

For the remainder of this section we let $D$ be a UFD, and assume that $\left\{p_{i}\right\}$ is a sequence of distinct primes. $S^{m}(M)$ will be factorial if and only if each symmetric power $S^{m}(M)$ has the f.d.p. Since for each $x \in S^{m}(M)$ there is a nonzero element $d \in D$ such that $d x \in S^{m}\left(F_{1}\right), S^{m}(M)$ will have the f.d.p. if and only if each nonzero element of $S^{m}\left(F_{1}\right)$ has a finite number of divisors when considered as an element of $S^{m}(M)$. Let $x=\left(a_{0}, \ldots, a_{m}\right)$ be a nonzero element of the free module $S^{m}\left(F_{1}\right)$. When considered as an element of $S^{m}\left(F_{n+1}\right)$, the only prime power divisors of $x$ are those it had as an element of $S^{m}\left(F_{n}\right)$ and, possibly, a power of $p_{n}$. (This follows from knowing the matrix for $S^{m}\left(F_{n}\right) \subseteq S^{m}\left(F_{n+1}\right)$.) Now the image of $x$ in $S^{m}\left(F_{n+1}\right)$ has each of its components divisible by $p_{n}$ except possibly the first one, $a_{0}-a_{1} x_{n}+\cdots+$ $a_{m}\left(-x_{n}\right)^{m}$. Hence $x$ will have a finite number of divisors as an element of $S^{m}(M)$ if and only if there is an integer $N$ such that $n \geqslant N$ implies $p_{n}+a_{0}-$ $a_{1} x_{n}+\cdots+a_{m}\left(-x_{n}\right)^{m}$. And from this we arrive at the conclusion that $S^{m}(M)$ is factorial if and only if for every nonzero polynomial $f(X) \in D[X]$ with $\operatorname{deg} f(X) \leqslant m$ there is an integer $N$ such that $n \geqslant N$ implies $p_{n} \nmid f\left(x_{n}\right)$. Similarly, $S(M)$ is factorial if and only if for every nonzero polynomial $f(X) \in$ $D[X]$ there is an integer $N$ such that $n \geqslant N$ implies $p_{n} \nmid f\left(x_{n}\right)$.

Let $Z$ be the ring of integers.

4.1. THEOREM. There is a rank two torsion-free abelian group $M$ such that $S_{Z}(M)$ is a UFD, but $M$ is not a submodule of any direct product of copies of $Z$.

Proof. Inductively choose a sequence of primes as follows. Let $p_{1}=2$. Assuming $p_{1}, \ldots, p_{n-1}$ have been chosen, let $x_{n}=1+p_{1}+p_{1} p_{2}+\cdots+$ $p_{1} \cdots p_{n-1}$ and choose a prime $p_{n}>n\left(1+x_{n}+\cdots+x_{n}^{n-1}\right)$. Then given a nonzero $f(X)=a_{0}+\cdots+a_{m} X^{m} \in Z[X]$, choose $N>\max \left\{m,\left|a_{0}\right|, \ldots,\left|a_{m}\right|\right\}$. 
If $n \geqslant N$ we have $\left|f\left(x_{n}\right)\right| \leqslant n\left(1+x_{n}+\cdots+x_{n}^{n-1}\right)<p_{n}$, and hence $p_{n} \nmid$ $f\left(x_{n}\right)$. It follows that $S(M)$ is a UFD, where $M$ is the module determined by $\left\{p_{i}\right\}$. The second assertion about $M$ (i.e., that it is not "torsionless") is proved in [7].

4.2. THEOREM. For each integer $s \geqslant 1$ there is a rank two torsion-free abelian group $M_{s}$ such that $S^{i}\left(M_{s}\right)$ is factorial for $0 \leqslant i<2^{s}$, but $S^{i}\left(M_{s}\right)$ is not factorial for $i \geqslant 2^{s}$.

Theorem 4.2 will be proved once we have carefully selected a sequence of primes. This careful selection of primes is number theoretic in nature and so we make it a separate theorem from which 4.2 follows.

4.3. THEOREM. Given a positive integer $s$, there is a sequence of distinct primes $\left\{p_{i}\right\}$ such that if $x_{n}=1+p_{1}+\cdots+p_{1} \cdots p_{n-1}$, then

(i) $p_{2 n+1} \mid 1+\left(x_{2 n+1}\right)^{2^{s}}$ for all $n \geqslant 0$, and

(ii) if $f(X) \in Z[X], f(X) \neq 0$, has degree less than $2^{s}$, then $p_{n} \mid f\left(x_{n}\right)$ for only finitely many $n$.

Proof. Choose $p_{1}=2$ and suppose $p_{1}, \ldots, p_{2 n-1}$ have been chosen. Choose a prime $q \equiv 1\left(\bmod 2^{s+1}\right)$ such that $q>\left(x_{2 n}\right)^{2^{s}}+1$, using Dirichlet's theorem on primes in arithmetic progressions. Note that -1 has a $2^{s}$ th root $(\bmod q)$. Let $w \in Z$ represent this root.

Let $z_{n}=p_{1} \cdots p_{n-1}$. Consider the polynomial $g(X)=1+$ $\left(x_{2 n}+z_{2 n} X\right)^{2^{s}}$. The congruence $g(X) \equiv 0(\bmod q)$ has a solution $x=$ $\left(-x_{2 n}+w\right) / z_{2 n}$. (Note that $q>z_{2 n}$, so $z_{2 n} \not \equiv 0$.) Furthermore, $g(0)=$ $\left(x_{2 n}\right)^{2^{s}}+1 \neq 0(\bmod q)$, so $x \neq 0(\bmod q)$. By Dirichlet's theorem again, there is a prime $p_{2 n}>2 n\left(1+x_{2 n}+\cdots+x_{2 n}^{2^{s}-1}\right)$ such that $p_{2 n} \equiv x(\bmod q)$. This picks $p_{2 n}$. Let $p_{2 n+1}=q$.

Note that $p_{2 n+1} \neq p_{2 n}$ and $p_{2 n}, p_{2 n+1}>p_{i}$ for $1 \leqslant i \leqslant 2 n-1$, so all the primes are distinct.

By our choice of $p_{2 n}, p_{2 n+1} \mid 1+\left(x_{2 n}+z_{2 n} p_{2 n}\right)^{2^{s}}=1+\left(x_{2 n+1}\right)^{2^{s}}$, so (i) is verified.

To verify (ii), let $f(X) \in Z[X], f(X) \neq 0$ and suppose $\operatorname{deg} f(X)<2^{s}$. Let $N$ be an integer surpassing the maximum of the absolute values of the coefficients of $f(X)$. Then if $2 n \geqslant N,\left|f\left(x_{2 n}\right)\right|<N\left(1+x_{2 n}+\cdots+x_{2 n}^{2^{s-1}}\right)<$ $p_{2 n}$, so $p_{2 n} \mid f\left(x_{2 n}\right)$ only if $f\left(x_{2 n}\right)=0$. Since $f(X)$ has only a finite number of roots, this occurs for finitely many $n$. We have now to see that $p_{2 n+1} \mid f\left(x_{2 n+1}\right)$ for only finitely many $n$. Keeping in mind that $\left(x_{2 n+1}\right)^{2^{s}} \equiv-1\left(\bmod p_{2 n+1}\right)$ for $n \geqslant 0$, the following lemma will complete the proof.

4.4. Lemma. Let $s \geqslant 0$ be an integer, $\left\{p_{i}\right\}$ be a sequence of distinct 
primes, and $\left\{x_{i}\right\}$ a sequence of integers. If $f(X) \in Z[X]$ has degree less than $2^{s}, x_{n}^{2^{s}} \equiv-1\left(\bmod p_{n}\right)$ for $n \geqslant 1$, and $f\left(x_{n}\right) \equiv 0\left(\bmod p_{n}\right)$ for $n \geqslant 1$, then $f(X)=0$.

Proof. We use induction on $s$, the result being clear if $s=0$. Let $f(X)$ $=\Sigma_{i=0}^{2^{s}-1} a_{i} X^{i}$. From $0 \equiv \Sigma a_{i} x_{n}^{i}\left(\bmod p_{n}\right)$ we get

$$
x_{n} \sum_{(i \text { odd })} a_{i}\left(x_{n}^{2}\right)^{(i-1) / 2} \equiv \sum_{(i \text { even })} a_{i}\left(x_{n}^{2}\right)^{i / 2}\left(\bmod p_{n}\right)
$$

Squaring both sides we obtain $x_{n}^{2} g^{2}\left(x_{n}^{2}\right) \equiv h^{2}\left(x_{n}^{2}\right)$, where $g, h$ are the polynomials in $x_{n}^{2}$ appearing in the preceding congruence. Now the highest power of $x_{n}^{2}$ which occurs in $g^{2}\left(x_{n}^{2}\right)$ or $h^{2}\left(x_{n}^{2}\right)$ is $\left(x_{n}^{2}\right)^{2^{s}-2} \equiv\left(x_{n}^{2}\right)^{2^{s-1}}\left(x_{n}^{2}\right)^{2^{s-1}-2} \equiv-\left(x_{n}^{2}\right)^{2^{s-1}-2}$ $\left(\bmod p_{n}\right)$. Hence $x_{n}^{2} g^{2}\left(x_{n}^{2}\right)-h\left(x_{n}^{2}\right)$ can be expressed as a polynomial $r\left(x_{n}^{2}\right)$ of degree less than $2^{s-1}$ in $x_{n}^{2}$. Since neither $g$ nor $h$ nor the relations used in reducing to $r\left(x_{n}^{2}\right)$ depend on $n$, the polynomial $r\left(x_{n}^{2}\right)$ does not depend on $n$. By the induction hypothesis, $r(X)=0$.

Now let $y$ be a complex $2^{s-1}$ th root of -1 . Then replacing $x_{n}^{2}$ by $y$ in the reduction above we get $y g^{2}(y)-h^{2}(y)=r(y)=0$. Hence $\sqrt{y} g(y)=h(y)$. Let $x=\sqrt{y}$. Then $x g\left(x^{2}\right)=h\left(x^{2}\right)$ yields $f(x)=0$. But $x$ is a $2^{s}$ th root of -1 , and so has minimal polynomial $X^{2^{s}}+1$. Thus $f(X)=0$.

ACKNOWLEDGEMENT. The author benefited from conversations with $G$. Keller and R. E. Stong during the writing of this paper.

\section{REFERENCES}

1. N. Bourbaki, Elements of mathematics, Commutative algebra, Chap. VII, Actualités Sci. Indust., no. 1314, Hermann, Paris; Addison-Wesley, Reading, Mass., 1965, 1972. MR 41 \# 5339; 50 \# 12997.

2. D. L. Costa, Symmetric algebras and retracts, Dissertation, Univ. of Kansas, 1974.

3. R. M. Fossum, The divisor class group of a Krull domain, Springer-Verlag, Berlin and New York, 1973.

4. L. Fuchs, Infinite abelian groups, Vol. II, Academic Press, New York, 1973. MR 50 \# 2362.

5. D. Lazard, Autour de la platitude, Bull. Soc. Math. France 97 (1969), 81-128. MR 40 \# 7310; erratum, 41, p. 1965.

6. A.-M. Nicolas, Modules factoriels, Bull. Sci. Math. (2) 95 (1971), 33-52. MR 44 \# 1653.

7. - Extensions factorielles et modules factorable, Bull. Sci. Math. 98 (1974), $117-143$.

8. P. Samuel, Anneaux gradués factoriels et modules réflexifs, Bull. Soc. Math. France 92 (1964), 237-249. MR 32 \# 4160.

DEPARTMENT OF MATHEMATICS, UNIVERSITY OF VIRGINIA, CHARLOTTESVILLE, VIRGINIA 22903 